\title{
Flash pyrolysis of silicon-bound hydrocarbons *
}

\author{
W.A. Hartgers *, J.S. Sinninghe Damsté and J.W. de Leeuw \\ Delft University of Technology, Faculty of Chemical Technology and Materials' Science, \\ Organic Geochemistry Unit, De Vries van Heystplantsoen 2, 2628 RZ Delft (The Netherlands)
} (Received October 2, 1990; accepted in final form December 7, 1990)

\section{ABSTRACT}

Curie-point flash pyrolysis-gas chromatography/mass spectrometry of $\mathbf{n}$-octadecane, $\mathrm{n}$-propylbenzene and $\mathrm{n}$-propylcyclohexane chemically linked to silica has been performed in order to gain a better understanding of the mechanism of flash pyrolysis by which hydrocarbons are generated from kerogens. The major products result via a 6 -membered ring rearrangement involving the $\mathrm{Si}-\mathrm{O}-\mathrm{C}$ linkage and from cleavage of $\mathrm{C}-\mathrm{C}$ bonds allylic to primary or secondary alkyl radicals.

Flash pyrolysis; gas chromatography; hydrocarbons; kerogens; mass spectrometry; pyrolysis.

\section{INTRODUCTION}

By far the greater part of organic carbon on Earth is present in kerogen (ca. $10^{22} \mathrm{~g}$ ), a high-molecular weight fraction of sedimentary organic matter not soluble in common organic solvents [1]. Upon thermal stress in the subsurface kerogens act as oil source rocks [2]. Recently, it was reported that kerogens partly originate from highly resistant biomacromolecules [3]. Upon flash pyrolysis all types of kerogen generate hydrocarbons in relatively high abundance. In many cases the pyrolysates are dominated by homologous series of $\mathbf{n}$-alkanes and $\mathbf{n}$-alkenes though complex mixtures of saturated and polycyclic aromatic compounds are clearly present as well [4].

The mechanisms by which these hydrocarbons are generated from the kerogen matrix or from the newly discovered, reasonably well-characterized highly aliphatic biomacromolecules is far from known [5]. Detailed studies of suitable macromolecular standard compounds are a prerequisite to a better understanding of the mechanisms by which hydrocarbons are generated from kerogens during "natural" and flash-pyrolysis.

ॠ Delft Organic Geochemistry Unit Contribution 236. 

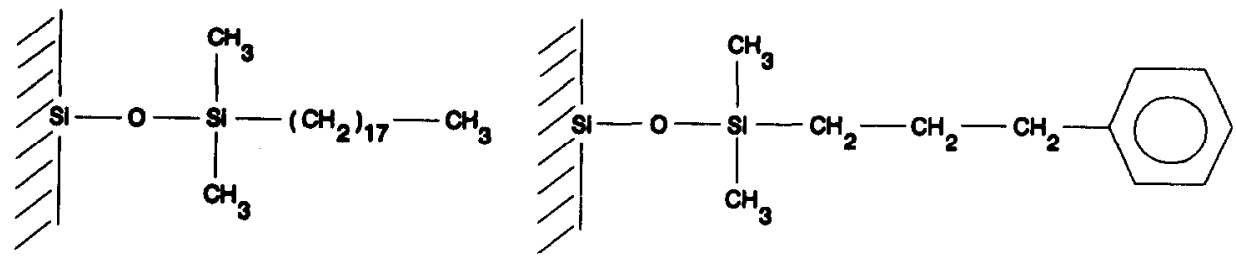

1

$\underline{2}$

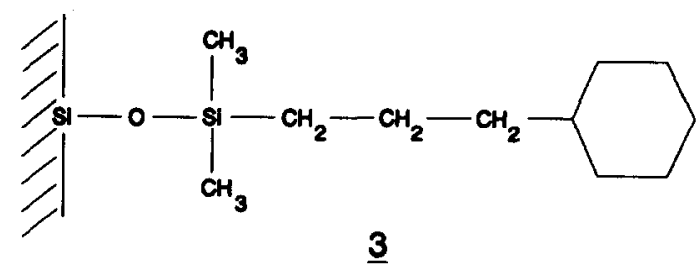

Fig. 1. Structures of silicon-bound n-octadecane (1), silicon-bound n-propylbenzene (2) and silicon-bound n-propylcyclohexane (3).

To this end a preliminary investigation was performed with the three model compounds shown in Fig. 1. Previous pyrolysis studies indicated that silicon-linked hydrocarbons are suitable model compounds to study mechanisms of hydrocarbon generation, because the silica backbone remains intact and does not interfere with the organic reaction products $[6,7]$.

\section{EXPERIMENTAL}

Silicon-bound octadecane (compound 1, Fig. 1) was commercially available as $\mathrm{C}_{18}$ /CORASIL (Waters Assoc.). The other reversed phase silica column materials (compounds 2 and 3 , Fig. 1) were kindly provided by the Analytical Section of the Faculty of Chemical Technology and Materials' Science of the TU Delft. Their syntheses involved a one-step catalytic hydrosilylation of the terminal double bond of 3-phenyl-1-propene and 3-cyclohexyl-1-propene with dimethylchlorosilylhydride $[8,9]$. This reaction favours the formation of the anti-Markovnikov-addition product dimethylalkylsilylchloride. This mono-functionalized sililizing agent was subsequently coupled to the free $\mathrm{OH}$-groups of activated silica in a dry atmosphere. The final product was washed thoroughly with methanol and dried for $12 \mathrm{~h}$ at $150^{\circ} \mathrm{C}$ in vacuo.

The samples were pressed on a flattened ferromagnetic wire according to the method described by Venema and Veurink [10]. The on-line flash pyrolysis was performed by inductive heating of the ferromagnetic wire $(\mathrm{Fe}$, Curie temperature $770^{\circ} \mathrm{C}$ ) in $0.15 \mathrm{~s}$ to its final temperature at which it was held for $10 \mathrm{~s}$. A Curie-point high frequency generator (Fischer, model 9425) 
was used to induce the magnetic field. The gas chromatograph (Varian 3700) was equipped with a cryogenic unit and programmed from $0^{\circ} \mathrm{C}(5 \mathrm{~min})$ to $300^{\circ} \mathrm{C}(10 \mathrm{~min})$ at a rate of $3^{\circ} \mathrm{C} / \mathrm{min}$. Separation of the products was achieved by a $25 \mathrm{~m}$ fused silica capillary column coated with chemically bound CP-Sil $5(0.32 \mathrm{~mm}$ I.D.; film thickness $0.45 \mu \mathrm{m})$. Helium was used as carrier gas. The temperature of the flame ionisation detector was $320^{\circ} \mathrm{C}$.

Flash pyrolysis-gas chromatography/mass spectrometry (Py-GC/MS) was performed using the same pyrolysis and GC-conditions as described above with a Hewlett Packard HP-5890 gas chromatograph coupled to the EI ion source of a VG-70s double focussing mass-spectrometer (mass range $m / z$ 40-800; cycle time $2 \mathrm{~s}$; ionisation energy $70 \mathrm{eV}$ ).

\section{RESULTS AND DISCUSSION}

Flash pyrolysis of silicon-bound hydrocarbons

The Py-GC traces of the silicon-bound hydrocarbons are shown in Fig. 2. The peaks were identified based on mass spectral data obtained via PyGC/MS analyses.

The Py-GC trace of silicon-bound n-octadecane (Fig. 2A) shows a series of $n$-alkanes and $n$-alkenes up to $C_{18}$. The octadecyl radical initially formed by homolytic cleavage of the relatively weak $\mathrm{Si}-\mathrm{C}$ bond [11] can stabilize to octadecane by an intermolecular H-radical transfer leading to the formation of a new radical R (Fig. 3). The lack of hydrocarbons with more than 18 carbon atoms indicates that the recombination of two radicals is an insignificant process. Scrambling of the primary radical leads to the formation of secondary radicals which can also stabilize via intermolecular $\mathrm{H}$-radical transfer to n-octadecane. An homologous series of n-alk-1-enes up to $\mathrm{C}_{17}$ is generated from these secondary radicals by cleavage of the allylic $\mathrm{C}-\mathrm{C}$ bond (Fig. 3). The other products from this reaction, primary radicals, are thought to stabilize by intermolecular $\mathrm{H}$-radical transfers resulting in a homologous series of $n$-alkanes up to $\mathrm{C}_{16}$. The occurrence of the scrambling process is supported by the relative enhancement of $n$-decene and $n$-undecene indicating the so-called back-bite phenomenon. The favoured formation of $n$-decene and $n$-undecene can be explained by two subsequent 1,5 hydrogen migrations starting from the initially formed primary $\mathrm{C}_{18}$ radical. A similar phenomenon is also observed during the pyrolysis of polyethene [12].

The intermolecular $\mathrm{H}$-radical transfer process leads to the formation of silicon-bound alkyl radicals, which will undergo the same type of stabilisation reactions as mentioned above (see Fig. 3). It is noteworthy that the relatively low abundance of $n$-heptadecane is a direct consequence of the above postulated mechanisms. 


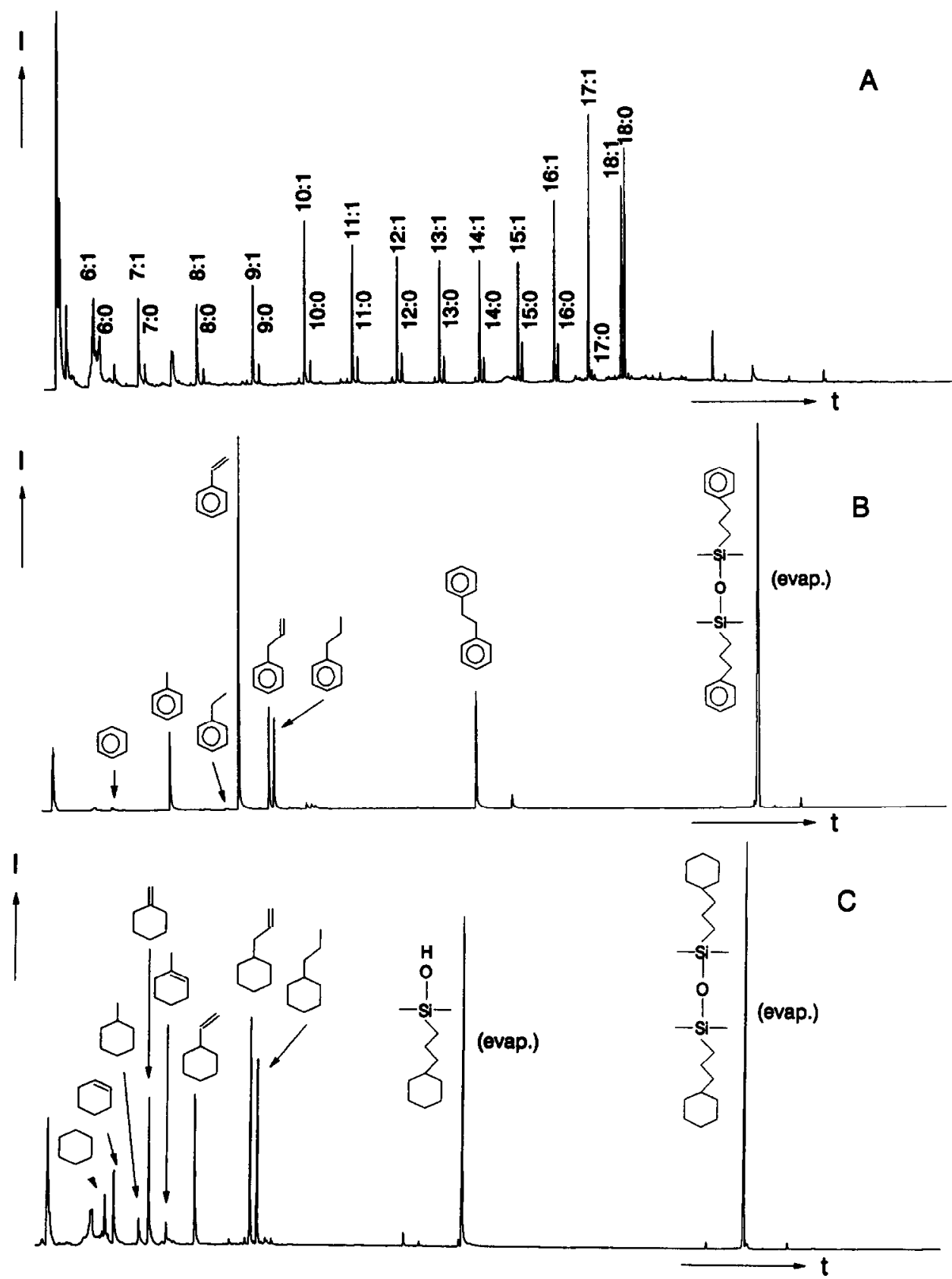

Fig. 2. Gas chromatograms of flash pyrolysates of: (A), silicon-bound n-octadecane; (B), silicon-bound n-propylbenzene; (C), silicon-bound n-propylcyclohexane.

n-Heptadecene, the most abundant compound in the flash pyrolysate, is supposed to be released from the macromolecule via a 6-membered ring rearrangement (Fig. 3). Obviously, the silicon-bound oxygen atom plays an important role in the electron transfer. The other proposed product of this 

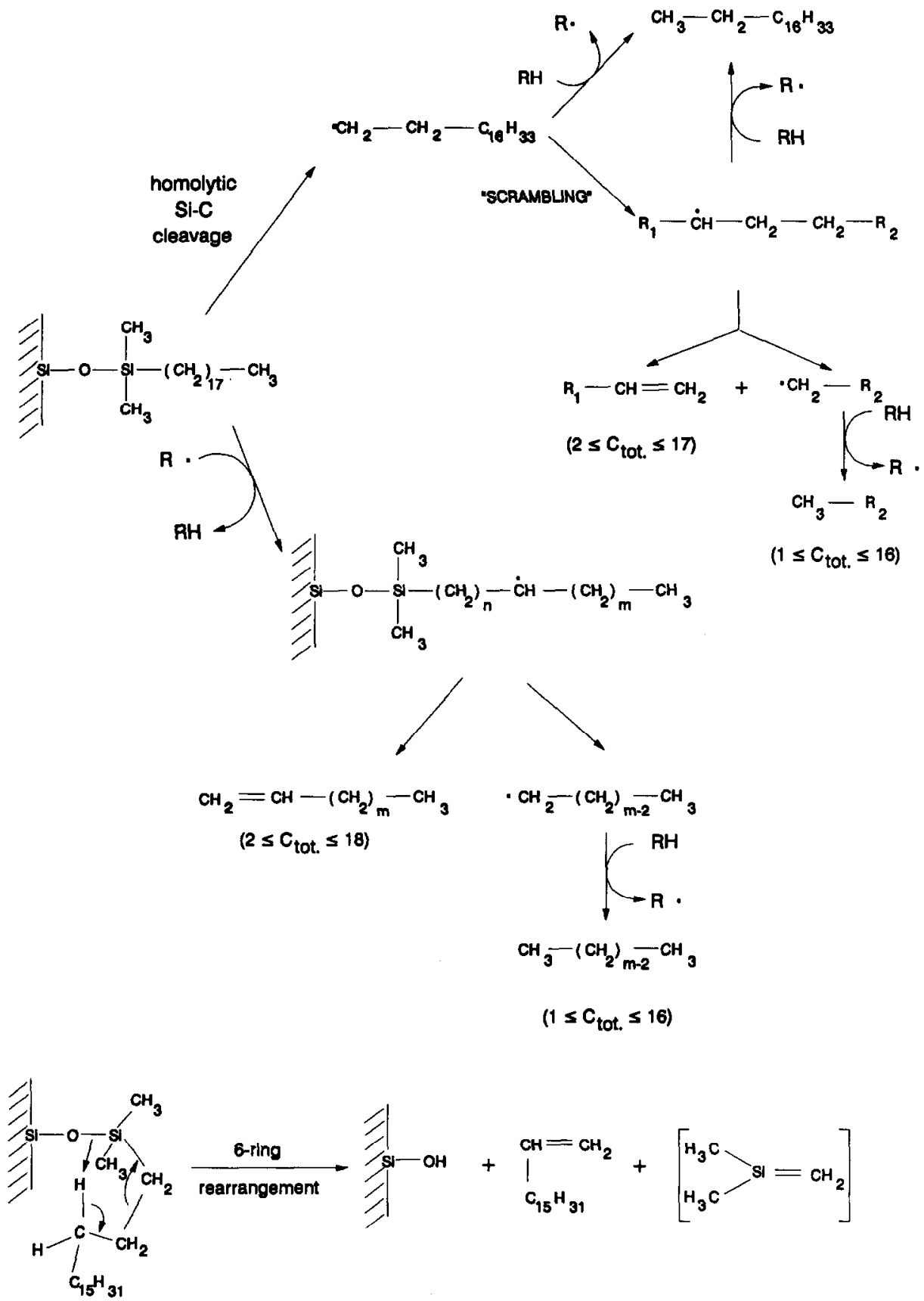

Fig. 3. Proposed mechanisms of pyrolysis of silicon-bound n-octadecane.

rearrangement, 1,1-dimethylsilaethene, was not detected by mass spectrometric analysis. It is supposed to react immediately with any nucleophile present, for example a Si-OH group, to yield trimethylsilylated silica as the stable end product [11]. 


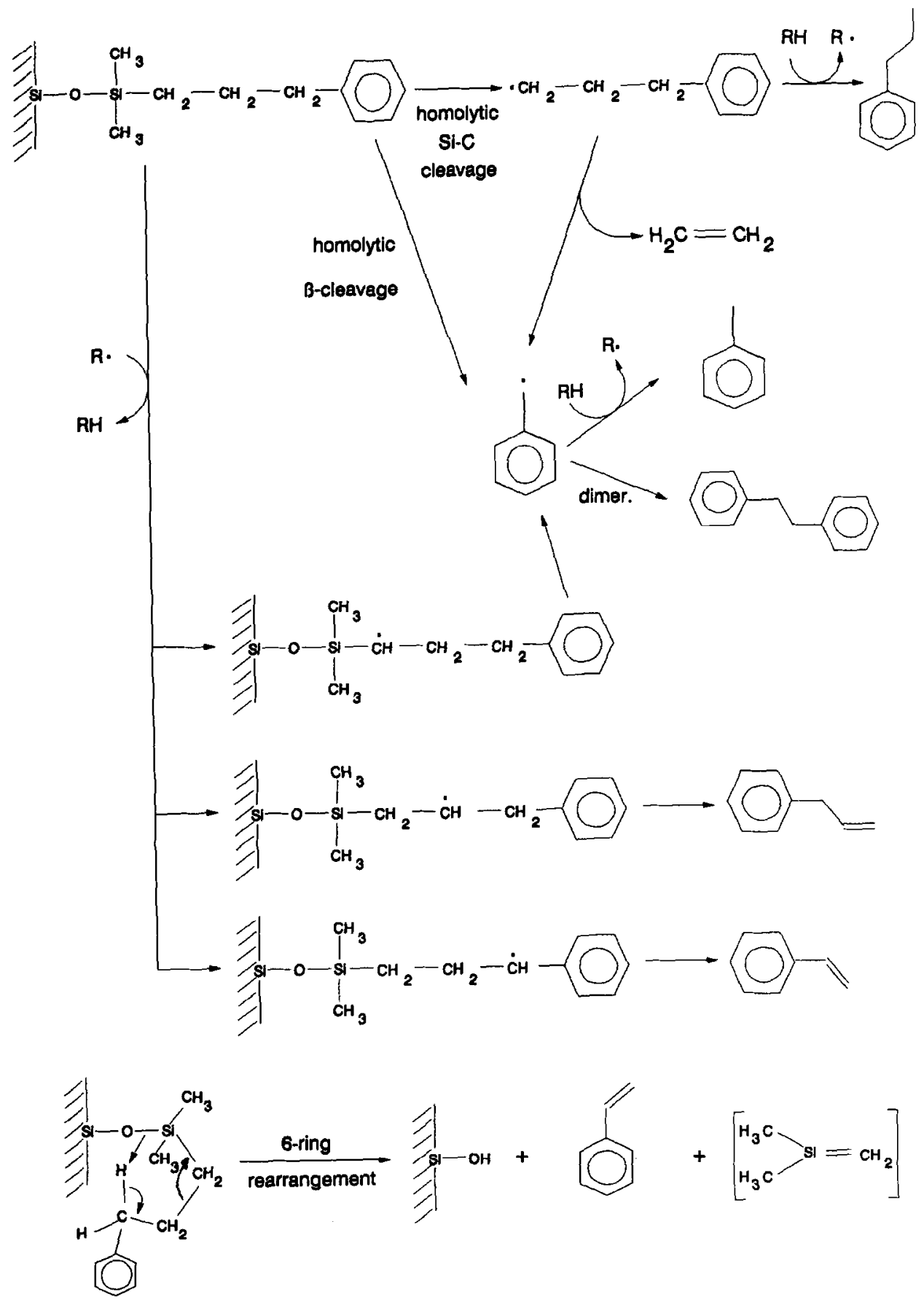

Fig. 4. Proposed mechanisms of pyrolysis of silicon-bound n-propylbenzene. 


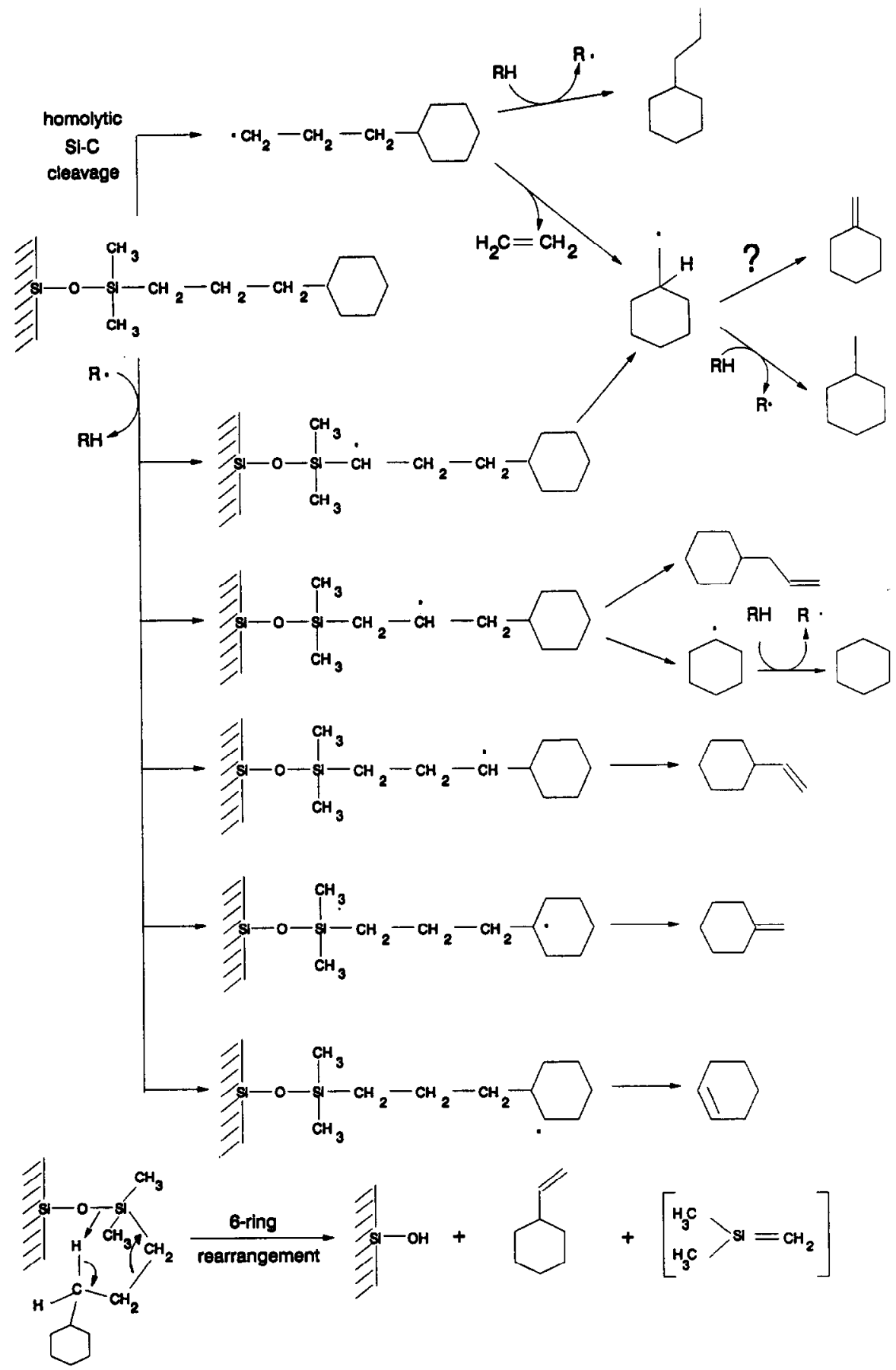

Fig. 5. Proposed mechanisms of pyrolysis of silicon-bound n-propylcyclohexane. 
Figure 2B shows the gas chromatogram of the flash pyrolysate of siliconbound n-propylbenzene. The last eluting silicon compound is not a pyrolysis product; it is also present in large quantities upon thermal extraction of the sample using a Curie temperature of $358^{\circ} \mathrm{C}$. The presence of this low-molecular-weight compound is due to a side reaction during the synthesis of the macromolecule. Despite the fact that the macromolecule was washed thoroughly and heated subsequently at $150^{\circ} \mathrm{C}$ in vacuo is is obviously still present in the sample. This is probably due to very strong adsorption of this side-product to the macromolecule.

The major pyrolysis product resulting from the 6-membered ring rearrangement mentioned above (cf. Figs. 3 and 4) is styrene. The other pyrolysis products result from allylic $\mathrm{C}-\mathrm{C}$ cleavages and intermolecular $\mathrm{H}$-radical transfers of both the primary radical generated by $\mathrm{Si}-\mathrm{C}$ cleavage and secondary radicals generated by $\mathrm{H}$-radical abstractions as indicated in Fig. 4. The formation of 1,2-diphenylethane is of interest. Dimerisation of the relatively stable benzylradical is proposed to explain this product because allylic $\mathrm{C}-\mathrm{C}$ cleavage within the benzylradical is highly unlikely.

Fig. $2 \mathrm{C}$ shows the gas chromatogram of the flash pyrolysate of the silicon-bound n-propylcyclohexane. A separate thermal extraction experiment at a Curie temperature of $358^{\circ} \mathrm{C}$ revealed that the silicon-containing compounds in the mixture are present as such and are probably side-products produced during the synthesis of the macromolecule (see above).

The formation of all pyrolysis products can be explained via the general mechanisms postulated for the other two silicon-bound hydrocarbons; allylic $\mathrm{C}-\mathrm{C}$ cleavage and intermolecular $\mathrm{H}$-radical transfer of the radical generated by $\mathrm{Si}-\mathrm{C}$ cleavage leads to $\mathrm{n}$-propylcyclohexane and via expulsion of ethene to methylcyclohexane. Stabilisation of the methylcyclohexane radical via abstraction of the $\alpha-\mathrm{H}$ radical may be taken into account because that hydrogen is attached to a tertiary carbon atom in this case (Fig. 5).

A variety of secondary radicals of the substrate can be generated via intermolecular hydrogen radical abstraction. In Fig. 5 only those secondary radicals are shown which form volatile pyrolysis products via allylic $\mathrm{C}-\mathrm{C}$ cleavages and subsequent stabilisation of primary radicals through intermolecular hydrogen radical transfers. These reactions will not release volatile products from other secondary radicals in the propylcyclohexane moiety.

The six-membered ring rearrangement reaction in this macromolecule results in the formation of ethenylcyclohexane (Fig. 5).

Application to kerogen pyrolysis

The mechanisms of pyrolysis reactions of alkyl moieties present in highmolecular-weight substances as postulated above can explain to some extent the hydrocarbon distributions in pyrolysates of kerogens and highly aliphatic 
biomacromolecules [4,5]. The often observed homologous series of n-alkanes and n-alk-1-enes are probably due to cleavage of a $\mathrm{C}-\mathrm{X}(\mathrm{X}=\mathrm{O}, \mathrm{S}$, etc.) bond followed by alkyl $\mathrm{C}-\mathrm{C}$ cleavage and $\mathrm{H}$-radical transfer reactions of the initially formed radicals or secondary radicals. Assuming that a large variety of alkyl moieties is present in kerogens it is virtually impossible to reconstruct the distribution patterns of alkyl moieties in these sedimentary substances when a complex mixture of hydrocarbons is already generated from one type of alkyl moiety, such as the $n-\mathrm{C}_{18} \mathrm{H}_{37}$ in $\mathrm{C}_{18}$-Corasil.

The results of the pyrolysis of the silicon-bound $n$-propylbenzene indicates that $\beta$-cleavage with respect to the aromatic unit is a favoured process although experiments with longer alkyl chains between the silicon and the aromatic unit will be necessary to demonstrate this. A $\beta$-cleavage with respect to aromatic units in kerogens may reveal the original distribution patterns of aromatic moieties and hence will yield information about the contributing bio(macro)molecules of kerogens [13].

Toluene is often a major pyrolysis product in kerogen pyrolysates. However, its dimer, 1,2-diphenylethane has not been encountered. This could mean that in kerogen pyrolysates numerous other relatively stable radicals are present for termination reactions or that high concentrations of H-radicals or hydrogen are simultaneously produced which convert the benzyl radical to toluene.

Flash-pyrolysis experiments with model compounds like the siliconbonded hydrocarbons in the presence of hydrogen or hydrogen donors are probably worthwhile in this respect. Such experiments are also of interest to verify if primary radicals can be stabilised (quenched) early on so that pyrolysates become less complex and more directly related to the structure of the macromolecules from which they are generated.

\section{ACKNOWLEDGEMENTS}

The authors wish to thank Mr. H.A.H. Billiet for providing the samples and Dr. A. Venema for making available to us an unpublished report. Mr. W. Pool and Mrs. A. Knol are acknowledged for technical support.

\section{REFERENCES}

1 B. Durand, Kerogen-Insoluble Organic Matter from Sedimentary Rocks, Editions Technip, Paris, 1980.

2 B.P. Tissot and D.H. Welte, Petroleum Formation and Occurrence, Springer, Heidelberg, 2nd edn., 1984.

3 E.W. Tegelaar, S. Derenne, C. Largeau and J.W. de Leeuw, Geochim. Cosmochim. Acta, 53 (1989) 3103. 
4 D. van de Meent, S.C. Brown, R.P. Philp and B.R.T. Simoneit, Geochim. Cosmochim. Acta, 44 (1980) 999.

5 E.W. Tegelaar, J.W. de Leeuw, C. Largeau, S. Derenne, H.R. Schulten, R. Müller, J.J. Boon, M. Nip and J.C.M. Sprenckels, J. Anal. Appl. Pyrolysis, 15 (1989) 29.

6 L. Hansson and L. Trojer, J. Chromatogr., 207 (1981) 1.

7 A. Venema, personal communication, 1990.

8 D. Seyferth and R.B. King (Eds.), Organometallic Chemistry Reviews; Annual Surveys; Silicon-Germanium-Tin-Lead, Elsevier, Amsterdam, 1978, p. 11.

9 G.E. Berendsen, K.A. Pikaart and L. de Galan, J. Liq. Chromatogr., 3 (1980) 1437.

10 A. Venema and J. Veurink, J. Anal. Appl. Pyrolysis, 7 (1985) 207.

11 E.W. Colvin, Silicon Reagents in Organic Synthesis, Academic Press, London, 1988, Chapter 1, p. 1.

12 T.P. Wampler and E.J. Levy, Analyst, 111 (1986) 1665.

13 J.S. Sinninghe Damsté, T.I. Eglinton, J.W. de Leeuw and P.A. Schenck, Geochim. Cosmochim. Acta, 53 (1989) 873. 